\title{
A Study on Diurnal Variation of Cosmic Ray flux using Daejeon and Jang Bogo Neutron Monitors
}

\author{
Jongil Jung ${ }^{1 *}$ \\ Chungnam National University \\ 99 Daehak-ro, Yuseong-gu, Daejeon 34134, Korea \\ E-mail: jongil85@cnu.ac.kr
}

\author{
Suyeon $\mathrm{Oh}^{2}, \mathrm{Yu} \mathrm{Yi}^{1}$, Paul Evenson ${ }^{3}$, Roger Pyle ${ }^{4}$, Geonhwa Jee ${ }^{5}$, Jeong-Han Kim ${ }^{5}$, \\ Changsup Lee ${ }^{5}$ \\ ${ }^{1}$ Chungnam National University, 99 Daehak-ro, Yuseong-gu, Daejeon, 34134, Korea \\ ${ }^{2}$ Chonnam National University, 77, Yongbong-ro, Buk-gu, Gwangju, 61186, Korea \\ ${ }^{3}$ University of Delaware, Newark, DE 19716, U.S.A. \\ ${ }^{4}$ Pyle Consulting Group, Inc.,100 Illinois Ave. Suite 200, St. Charles, IL 60174, U.S.A. \\ ${ }^{5}$ Korea Polar Research Institute, 26 Songdomirae-ro, Yeonsu-gu, Incheon 21990, Korea
}

\begin{abstract}
The diurnal variation of cosmic ray flux is measured by ground neutron monitor. The local time of maximum cosmic ray flux is dependent on latitudinal location. Depending on solar activity, drift and diffusion effect control the local time of maximum cosmic ray flux (Oh et al., 2010). Recently, we installed two neutron monitors at Daejeon in South Korea (October 2011) and at Jang Bogo station in Antarctica (December 2015). In this study, we investigate the diurnal variation at two neutron monitors as the characteristics of two neutron monitors. The diurnal variations are analysed by harmonic analysis and pile-up method (Oh et al., 2010). In this report, we present the results on the diurnal variation of cosmic ray flux at Daejeon and Jang Bogo neutron monitors. We also report the process of neutron monitor installation at Jang Bogo station. We expect that two neutron monitors play an important role to provide the information on space weather as the member of neutron monitor network.
\end{abstract}

35th International Cosmic Ray Conference - ICRC2017

10-20 July, 2017

Bexco, Busan, Korea

\footnotetext{
$1 *$ Speaker

(c) Copyright owned by the author(s) under the terms of the Creative Commons 


\section{Introduction}

Cosmic rays are composed mainly of galactic cosmic ray (GCR) and solar cosmic ray (SCR). GCR particles come from outside solar system, which is thought to have originated form supernova remnants in the Milky Way and other galaxies. SCR particles originate from solar eruptions such as corona mass ejections and solar flares. Cosmic rays mostly consist of proton and helium particles [1]. Proton and helium do not directly come in the Earth's surface. These particles turn into secondary cosmic ray particles and then enter the Earth's surface. Secondary cosmic ray particles are generated by collisions between air molecules and cosmic ray particles in Earth's atmosphere. The secondary cosmic rays can reach the Earth's surface in the various particles such as neutrons, muons and gamma rays. Cosmic ray flux shows the anti-correlation with the sunspot number and 11-year cyclic variation [2]. It also shows the diurnal variation.

The diurnal variation of cosmic ray flux on the Earth has the sinusoids with amplitude of 1$2 \%$ flux. This phenomenon is related to diffusion of spiral direction and convection in the opposite direction of the Sun. Because of an asymmetry of geomagnetic field, the cutoff rigidity has different values for the Earth's geographic latitude. The cosmic ray fluxes have the maximum value at early afternoon and the minimum value at after mid-night in local time sector [3], [4]. The diurnal variation of cosmic ray flux can be measured by neutron monitors.

Neutron monitor is an instrument that measures the neutrons as the secondary cosmic rays on ground base. Neutron monitors have been collecting the data since 1948, during half a century, to start detecting IGY type neutron monitor. The IGY type neutron monitor was designed by John A. Simpson, firstly in 1948 [5]. Currently, NM64 type neutron monitors are popular and are installed at about 50 neutron monitor stations around the world.

In this study, we confirm the diurnal variation of cosmic ray flux at two Korean neutron monitors focusing on the time of maximum cosmic ray intensity. Also, we introduce two Korean neutron monitors and report the process of neutron monitor installation at Jang Bogo station.

\section{Neutron Monitors in Korea}

\subsection{Neutron Monitor at Daejeon}

Daejeon neutron monitor was installed in Daejeon, Korea in October 2011. It is located at Korea Research Institute of Standards and Science (KRISS; $36.39^{\circ} \mathrm{N}, 127.37^{\circ} \mathrm{E}$ ). Its height is 200 $\mathrm{m}$ and it has the cutoff of $11.2 \mathrm{GV}$. Daejeon neutron monitor is NM64 type and has a total of 18 tubes (called "18-tube NM64" neutron monitor) [6]. The NM64 neutron monitor mainly consists of three components, the reflector, the lead producer, and the moderator. The reflector moderates incoming neutron energy and reflects naturally occurring neutrons. The lead producer enhances moderation of neutrons and the moderator drives neutron particles with the state of almost $<1 \mathrm{eV}$ to optimize the capture cross section of neutrons. Inside the moderator, there is a counter filled with 10BF3 gas and neutrons are detected in here. The reflector and the moderator are made of polyethylene. Because of high cutoff, the Daejeon neutron monitor can provide the cosmic ray spectrum at high energy part. The data of Daejeon neutron monito will be soon published at Neutron Monitor Database (NMDB). 


\subsection{Neutron Monitor at Jang Bogo Station}

Jang Bogo neutron monitor was installed in Jang Bogo station, Antarctica in December 2015. The Jang Bogo station is Korea's second Antarctic research. It is located at the coast $\left(74.62^{\circ} \mathrm{S}\right.$, $164.23^{\circ} \mathrm{E}$ ) of Terra Nova Bay in Northern Victoria Land and the first Korean science station in Antarctica. The Jang Bogo neutron monitor's height is $26 \mathrm{~m}$ and it has the cutoff of almost 0.1 GV. The Jang Bogo station allows the easy access from the center of Antarctica and from the coast. The location could serve as a research base for the studies on climate change, topographical and geological survey, the upper atmosphere, and space science to enable the acquisition of various data and characterized researches.

Presently, we transferred only one unit (6-tubes) from McMurdo station. The rest units will be installed in December 2017 - March 2018. Jang Bogo and McMurdo stations are quite close each other geographically and geomagnetically. Therefore, it is expected that the observational results at McMurdo could be reproduced at Jang Bogo [7].

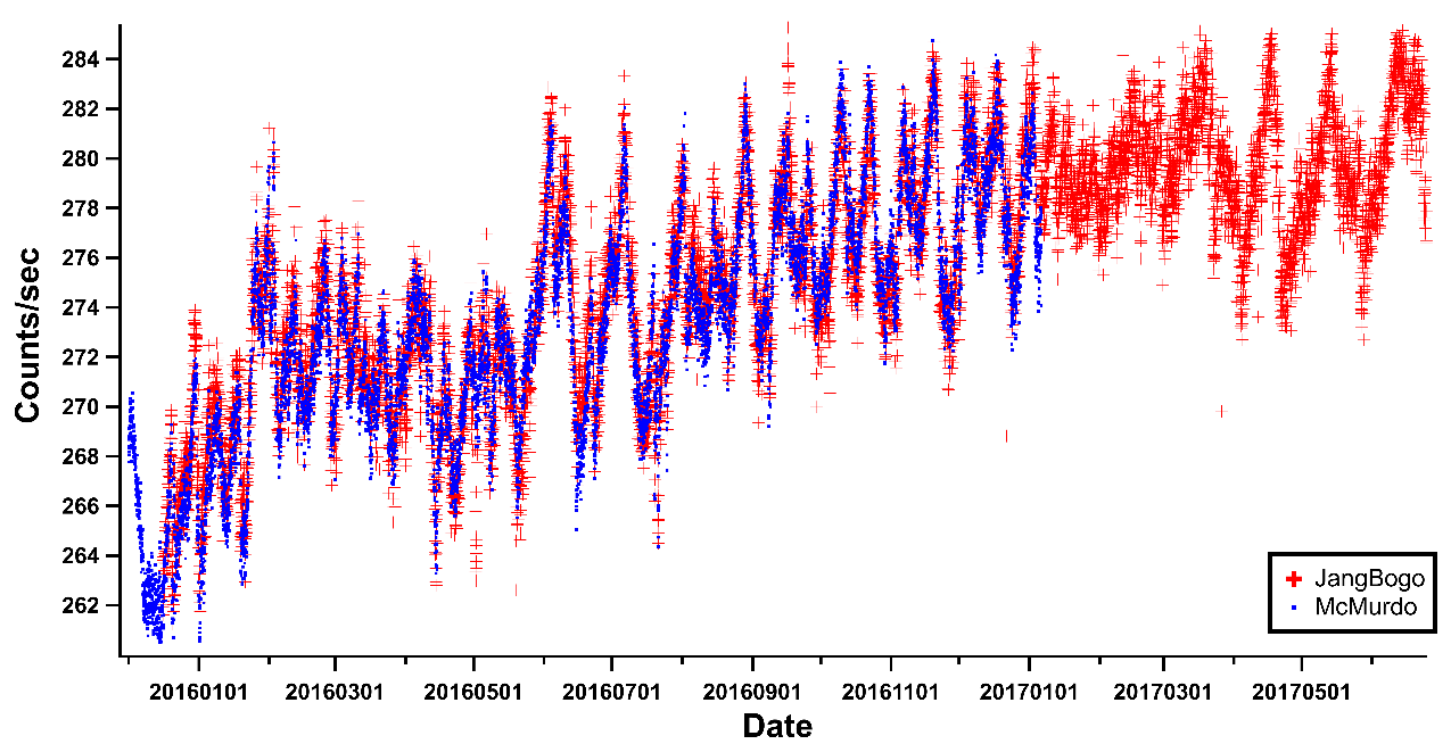

Fig. 1. Comparision of data between Jang Bogo and McMurdo neutron monitors. McMurdo neutron monitor was closed on 6 Jan. 2017.

Fig. 1 shows the comparison of neutron monitor data at Jang Bogo and McMurdo since Jan. 2016. The symbols of plus and dot indicate the data from Jang Bogo and McMurdo neutron monitors. The counts recorded at Jang Bogo and McMurdo neutron monitors show almost same values. McMurdo neutron monitor was closed on 6 Jan. 2016. Currently Jang Bogo neutron monitor provides the data at NMDB as the successor of McMurdo neutron monitor.

\section{Diurnal variation at Korean Neutron Monitors}

We apply the pile-up method [4] and harmonic analysis. The pile-up method performs the harmonic analysis one time for averaged cosmic ray flux in hourly distribution. When apply the pile-up method, we use the raw hourly data, 3-hour, and 5-hour smoothed data. We analyzed the data for the periods of 1 Jan. 2016 - 30 Jun. 2017 at Jang Bogo neutron monitor and of 11 Apr. 
2017 - 22 Jun. 2017 at Daejeon neutron monitor. We delete the data of dates on Forbush decreases and ground level enhancements. For instance, we removed the data of dates on the two weak Fobush decreases (1-3 May 2016: 3.0\% variation, 19-24 July 2016: 2.5\% variation) from Jang Bogo nueton monitor data.

Fig. 2 shows the diurnal variation at Jang Bogo neutron monitor. The $\mathrm{x}$ and $\mathrm{y}$ axas show the local time (LT) and the neutron count (counts per second) for Jang Bogo neutron monitor, respectively. The dotted, dashed, blue solid and red solid lines represents the results by pile-up method using raw, 3-hour smoothed, 5-hour smoothed data and by harmonic analysis. Each symbol on the line indicates the time of maximum cosmic ray flux for each result. For example, the symbol of cross (X) shows the time of maxim cosmic ray flux by harmonic analysis. The time of maximum cosmic ray flux by harmonic analysis is eariler than that by harmonic analysis.

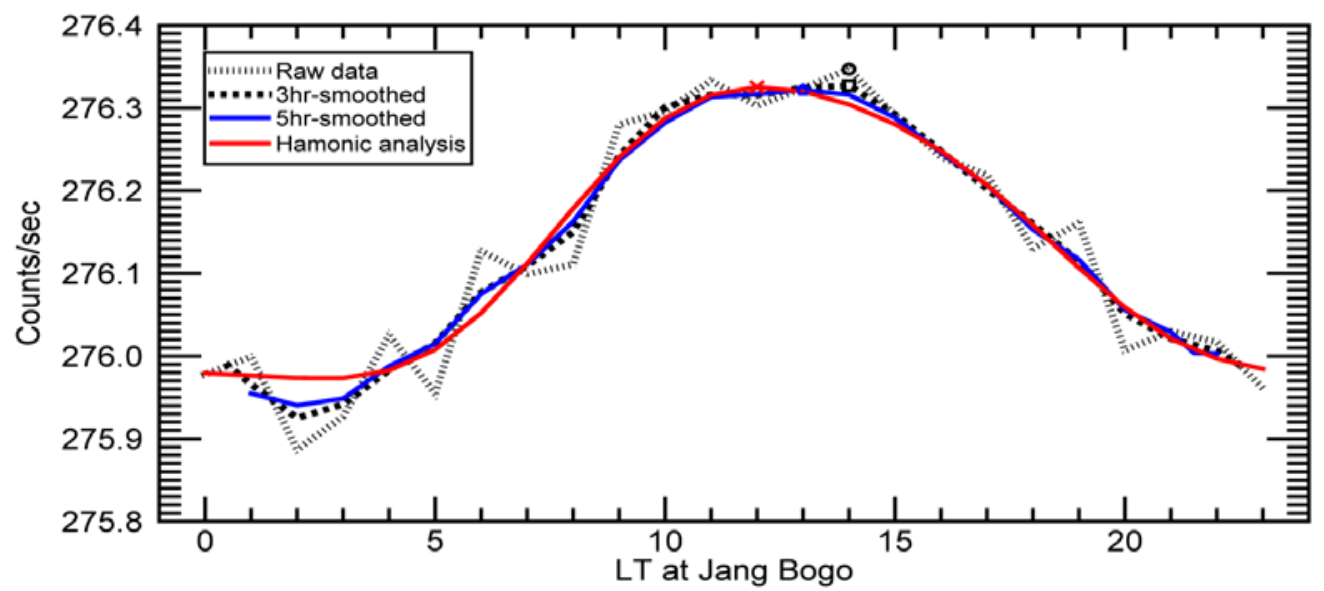

Fig. 2. The diurnal variation at Jang Bogo neutron monitor analyzed by pile-up method and harmonic analysis. The symbol indicates the time of maximum cosmic ray flux on each line by different methods.

Table 1. The time of maximum cosmic ray flux at Jang Bogo and Daejeon neutron monitors.

\begin{tabular}{|c|c|c|c|c|}
\hline \multirow{2}{*}{ NM } & \multicolumn{3}{|c|}{ Pile-up method } & \multirow{2}{*}{ Harmonic analysis } \\
\cline { 2 - 4 } & Raw data & 3-hr smoothed & 5-hr smoothed & \\
\hline Jang Bogo & 14 LT & 14 LT & 13 LT & 12 LT \\
\hline Daejeon & 13 LT & 13 LT & 13 LT & 14 LT \\
\hline
\end{tabular}

Fig.3 shows the diurnal variation at Daejeon neutron monitor. The display of Fig. 3 is the same as in Fig. 2. The time of maximum cosmic ray flux by harmonic analysis is later than that by pile-up method by one hour. The results of diurnal variation at two neutron monitor are summaried in Table 1. 


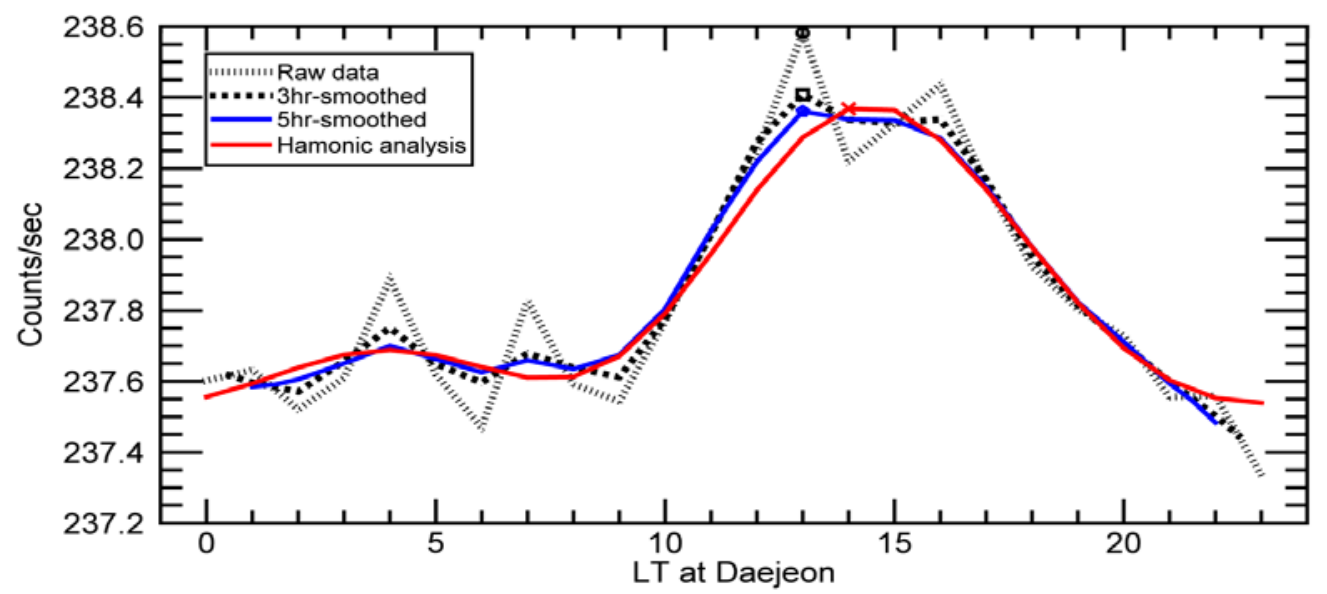

Fig. 3. The diurnal variation at Daejeon neutron monitor analyzed by pile-up method and harmonic analysis. The symbol indicates the time of maximum cosmic ray flux on each line by different methods.

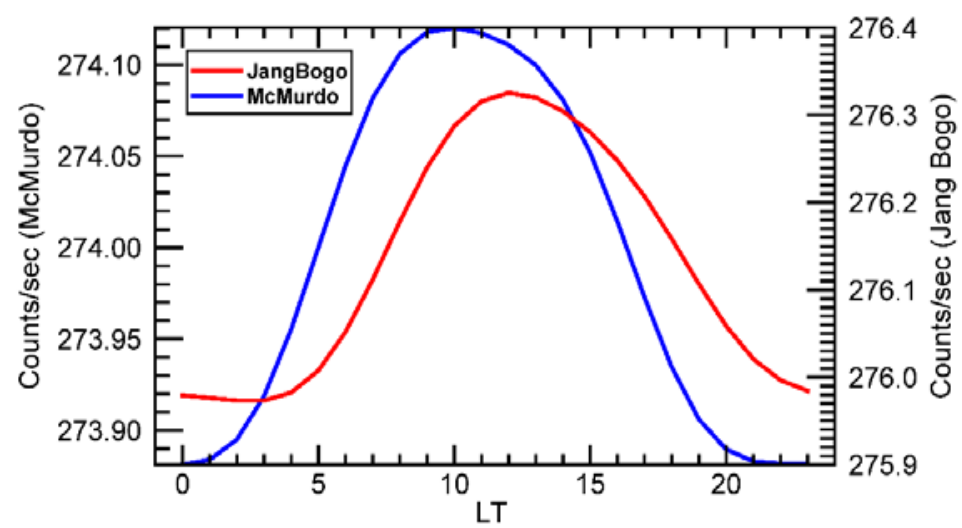

Fig. 4. The diurnal variation at Jang Bogo and McMurdo neutron monitors analyzed by harmonic analysis.

Fig. 4 shows the diurnal variation at Jang Bogo (red line) and McMurdo (blue line) neutron monitors during the periods of 1 Jan. 2016 - 30 Jun. 2017 at Jang Bogo and of 1 Jan. 2016 - 31 Dec. 2016 at McMurdo. This result is made by harmonic analysis. Two neutron monitors have two hours difference in the time of maximum cosmic ray flux. The time of maximum cosmic ray flux shows at 10 LT for McMurodo neutron monitor and at 12 LT for Jang Bogo neutron monitor. The diurnal variations at two neutron monitors also show the difference in the trend of variation such as width of phase. As mentioned in the previous section, two neutron monitor have the geographic and geomagnetic proximitities. We surmise that the difference in diurnal variation between two neutron monitors results from the stabilization state of Jang Bogo neutron monitor. In fact, Jang Bogo neutron monitor has experienced several power outages over the past year. 
Because it consists of only six tubes, the data recorded at Jang Bogo neutron monitor have the probabilty of statistical uncertainty. This issue can be solved after complete transportation of all tubes from McMurdo.

\section{Summary and Conclusions}

Korea has been operating two neutron monitors since 2011. One is located in Daejeon, Korea and the other is located in Jang Bogo station, Antartica. The latter is the succssion of McMurdo neutron monitor. We examine the diurnal variation at two neutron monitors as the characteristics of two neutron monitors. The diurnal variations are analysed by harmonic analysis and pile-up method. The analzed data are the periods of 1 Jan. 2016 - 30 Jun. 2017 for Jang Bogo and of 11 Apr. 2017 - 22 Jun. 2017 for Daejon neutron monitor.

At Jang Bogo neutron monitor, the time of maximum cosmic ray flux has the difference of two hours between results by two methods. With the respect to comparison with McMurdo neutron monitor, the harmonic analysis shows the reliable result by two-hour difference as shown in Fig. 4. The time of maximum cosmic ray flux has the difference of one hour between results by two methods at Daejeon neutron monitor.

We have to solve the difference in the diurnal variation between Jang Bogo and McMurdo neutron monitors. We guess that Jang Bogo neutron monitor has experienced the stabilization state since installation. But we expect that this issue can be solved after complete transportation of all tubes from McMurdo. The complete installation of all tubes can remove the probabilty of statistical uncertainty. We expect that two Korean neutron monitors can play an important role to provide the information on space weather as the member of neutron monitor network.

\section{References}

[1] J. A. Simpson, Elemental and Isotopic Composition of the Galactic Cosmic Rays, Annu. Rev. Nucl. Part. Sci. 33, 323 (1983).

[2] S. E. Forbush, World-wide cosmic-ray variations, 1937-1952, J. Geophys. Res., 59, 525 (1954).

[3] S. Y. Oh, Y. Yi, Local Times of Galactic Cosmic Ray Intensity Maximum and Minimum in The Diurnal Variation, J. Astron. Space Sci. 22(2), 117 (2007).

[4] S. Y. Oh, Y. Yi, J. W. Bieber, Modulation Cycles of Galactic Cosmic Ray Diurnal Anisotropy Variation, Solar Phys. 262, 199 (2010).

[5] J. A. Simpson, The Cosmic Ray Nucleonic Component: The Invention and Scientific Uses of the Neutron Monitor, Space Sci. Rev. 93, 11 (2000).

[6] S. Kang, et al., Characteristics of the 18-tube NM64-type Daejeon Neutron Monitor in Korea, J. Korean Phys. Soc. 61(5), 720 (2012).

[7] J. Jung, et al., Installation of Neutron Monitor at the Jang Bogo Station in Antarctica, J. Astron. Space Sci. 33(4), 345 (2016). 